\title{
Efficacy of Indirect Decompression in Lumbar Spinal Stenosis with Stand-alone Oblique Lateral Interbody Fusion
}

Chen Liu

Yijishan Hospital of Wannan Medical College https://orcid.org/0000-0002-8924-0582

Quanlai Zhao

Yijishan hopital

Yu Zhang

Yijishan hospital

Liang Xiao

Yijishan hospital

Xin Ge

Yijishan hospital

Hongguang Xu ( $\nabla$ xuhgyjsyy@163.com )

Research article

Keywords: Stand-alone OLIF, Lumbar spinal stenosis, Disc Height, Foraminal Height, Lumbar lordosis

Posted Date: March 11th, 2020

DOI: https://doi.org/10.21203/rs.3.rs-16687/v1

License: (9) This work is licensed under a Creative Commons Attribution 4.0 International License. Read Full License 


\section{Abstract}

\section{Background}

Oblique lateral interbody fusion (OLIF) has been gained more and more attention in the treatment of degenerative lumbar disease. The goal of this study was to evaluate the effect of indirect decompression in lumbar spinal stenosis with stand-alone OLIF.

\section{Methods}

Sixty-three patients with lumbar spinal stenosis who underwent stand-alone OLIF between July 2017 and May 2018 our department were included. Clinical outcomes including visual analogue scale (VAS) and Oswestry Disability Index (ODI) were recorded. Radiographic outcomes comprising of disc height (DH), foraminal height $(\mathrm{FH})$ and lumbar lordosis $(\mathrm{LL})$ were measured. Intraoperative data and complications were collected. All the data were compared preoperatively and postoperatively.

\section{Results}

Eighty-two segments were fused in sixty-three patients using stand-alone OLIF. The average follow-up time was $21.9 \pm 3.5$ months (range from 16 to 28 months). The $\mathrm{DH}$ increased from $0.9 \pm 0.3 \mathrm{~cm}$ preoperatively to $1.3 \pm 0.2 \mathrm{~cm}$ postoperatively, and the final follow-up was $1.1 \pm 0.2 \mathrm{~cm}(\mathrm{P}<0.01)$. The FH increased from $1.7 \pm 0.3 \mathrm{~cm}$ before surgery to $2.3 \pm 0.3 \mathrm{~cm}$ after surgery, but decreased to $2.1 \pm 0.3 \mathrm{~cm}$ at final follow-up $(P<0.01)$. The $L L$ increased from $38.0^{\circ} \pm 15.6^{\circ}$ before surgery to $42.7^{\circ} \pm 13.0^{\circ}$ at the final follow-up $(p<0.01)$. The VAS and ODI scores of all patients significantly improved at the final follow-up $(p<0.01)$. The total complication rate was $30.2 \%$. Only three patients received revision of posterior decompression and pedicle screw fixation.

\section{Conclusions}

Stand-alone OLIF is an effective option in selected patients with lumbar spinal stenosis.

\section{Background}

Lumbar spinal stenosis cause a series of clinical symptoms such as low back pain and intermittent claudication, which resulted from compression from either soft or calcified tissue of nerves and blood vessels in the spinal canal and neural foramen ${ }^{[1]}$. Serious cases may lead to loss of self-care ability and significantly affect the quality of life of patients ${ }^{[2]}$. It is believed that decompression with or without fusion is primary indication and goal for the treatment of lumbar spinal stenosis. Traditionally direct decompression and fusion for lumbar spinal stenosis include posterior lumbar interbody fusion (PLIF) and transforaminal lumbar interbody fusion (TLIF). However, paravertebral muscle detachment, nerve root retraction and manipulation of the dura could result in obvious iatrogenic complications ${ }^{[3]}$. Anterior lumbar interbody fusion (ALIF) with indirect decompression of the neural elements is now also regarded 
as a common procedure for lumbar spinal stenosis by restoring the height of intervertebral space ${ }^{[4]}$. Anterior surgery also brings a series of risks such as iliac vascular injury, abdominal contents injury, ureteral injury, retrograde ejaculation and so on ${ }^{[5]}$.

In recent years, the direct lateral interbody fusion/extreme lateral interbody fusion (DLIF/XLIF) as minimally invasive surgical method achieved excellent clinical and radiographic results for the treatment of lumbar spinal stenosis ${ }^{[6-8]}$. But the injury of psoas major and lumbar plexus nerve is inevitable. It was reported that the incidence of injury of psoas major muscle and lumbar plexus nerve is still high even with the help of neuromonitoring during the surgery ${ }^{[9]}$. Silvestre et al ${ }^{[10]}$ firstly reported oblique lateral interbody fusion (OLIF) in 2012, which could access the disc between anterior vessels and psoas major and establish working channels to handle the intervertebral space. OLIF has shorter operation time, larger fusion area and less nerve interference comparing to the posterior and anterior surgery. In addition, the contact area between the OLIF cage and the endplate is much larger with the cage spanning the lateral borders of the ring apophysis, which allows for the intervertebral space stretching to the maximum extent and the effective indirect decompression of the spinal canal and foramen. Up to now, there are few studies on stand-alone OLIF in the treatment of lumbar spinal stenosis. The goal of this study is to evaluate the efficacy of clinical and radiographic outcome and related complications of indirect decompression using a retrospective review of 63 patients with lumbar spinal stenosis who underwent stand-alone OLIF in our institution.

\section{Methods}

\section{Inclusion and Exclusion Criteria}

Inclusion Criteria were as follows: 1) patients who were diagnosed as lumbar spinal stenosis with grade A-C according to Schias's study ${ }^{[11]}$; 2) segments of lumbar spinal stenosis were located from L2 to $L 5 ; 3$ ) patients who experienced chronic low back pain and radiating pain to the lower extremities and could not be effectively treated conservatively for at least 3 months. Exclusion Criteria were: 1) patients with lumbar spinal stenosis were regarded as grade $D ; 2$ ) the facet joint was fused spontaneously according to the CT scan; 3 ) patients with osteoporosis (the T value of bone density tested by DXA was less than -2.5 ); 4) patients who were diagnosed with traumatic injuries, tumors, or infections of lumbar.

At last, a cohort of 63 patients from department of spine surgery, Yijishan hospital of Wannan Medical College between May 2017 and July 2018 were enrolled in the study.

\section{Stand-alone OLIF Procedure}

All the patients underwent general anesthesia and were placed in the stand right side position. The single segment transverse skin incision was in the same horizontal plane at the left lateral abdomen. For multilevel cases, the incision was in the middle of the surgical levels. All the incision was $4 \mathrm{~cm}$ long. The 
abdominal wall muscles were bluntly dissected. The psoas major was retracted posteriorly and the abdominal vessels were retracted anteriorly. Sequential dilators and retractor were placed over the a guide needle after it was correctly inserted in the middle of the target intervertebral disc with the help of a Carm. Then the operation field was exposed. The intervertebral disc was removed and the cartilage endplates were resected for exposure of the bony endplates. A wide and lordotic intervertebral fusion cage (Clydesdale; Medtronic Sofamor Danek, Memphis, Tennessee, USA)packed with allograft bone was inserted into the target disc with the guidance of $\mathrm{C}$-arm. In the end, the incision was sutured. All patients have not been received posterior internal fixation at one stage and no drainage tube was placed after surgery (Fig. 1).

\section{Clinical And Radiographic Evaluation}

Clinical outcomes were evaluated using a Visual analogue scale (VAS) score and the Oswestry disability index (ODI) before surgery and the final follow-up for comparison. Neutral anterior-posterior and lateral lumbar X-ray were used to assess radiographical results. Mean disc height ( $\mathrm{DH}=$ mean value of the leading and trailing edge height of the intervertebral disc), mean foraminal height ( $\mathrm{FH}=$ the distance from the lower position of the pedicle of upper veterbral and the upper position of the pedicle of the lower veterbral) were recorded before the operation, after the operation and at the final follow-up. And lumbar lordosis (LL) was measured before surgery and at the final follow-up. All the measurements were taken by three physicians specialized in spinal surgery, and the average values were used for analysis. The measuring method is shown in Fig. 2.

\section{Complications}

Complications were recorded during the operation and follow-up periods.

\section{Statistical analysis}

All data were statistically processed using SPSS 19.0 software (IBM Corp, Armonk, NY). The measurement data were expressed with mean \pm standard deviation. The t-test was performed on the VAS scores, ODI index and LL to compare the preoperative state with the final follow-up state, while analysis of variance was used for the comparison of data in $\mathrm{DH}$ and $\mathrm{FH}$. It was considered statistically different when $P$ value was less than 0.05 .

\section{Results}

\section{Patient Demographics and Operative Data}

The demographic data and treatment information of the patients are provided in Table 1. 


\section{Clinical And Radiographical Results}

The VAS score $(2.7 \pm 0.7$ points) and ODI index $(12.9 \% \pm 2.1 \%)$ at the final follow-up were both significantly lower compared to those observed before operation (VAS score: $4.8 \pm 1.1$ points; ODI: $30.6 \% \pm 6.0 \%$ ) $(p<$ 0.01 ) (Fig. 3). The radiographical results were assessed by DH and $\mathrm{FH}$ before the operation, after the operation and the final follow-up. The DH increased from $0.9 \pm 0.3 \mathrm{~cm}$ before the operation to $1.3 \pm 0.2 \mathrm{~cm}$ after the operation, and the final follow-up was $1.1 \pm 0.2 \mathrm{~cm}(p<0.01)$ (Fig. 4A). Besides, The FH increased from $1.7 \pm 0.3 \mathrm{~cm}$ preoperative to $2.3 \pm 0.3 \mathrm{~cm}$ postoperative, but decreased to $2.1 \pm 0.3 \mathrm{~cm}$ at final follow-up (Fig. 4B). The LL increased from $38.0^{\circ} \pm 15.6^{\circ}$ before the surgery to $42.7^{\circ} \pm 13.0^{\circ}$ the final follow-up $(p<0.01)$ (Fig. 5).

\section{Intraoperative Complications}

There were four cases (6.3\%) with intraoperative complications (Table 2). One patient (1.6\%) suffered from intraoperative vascular injury. Titanium clips were implanted after bipolar electrocoagulation hemostasis. No active hemorrhage was found after the operation. Two cases (3.2\%) suffered from endplate injury during the operation, who were not fixed by posterior internal fixation. These patients were protected by thoracolumbar braces. One patient $(1.6 \%)$ suffered peritoneal injury during the operation. The general surgeon was invited on the stage and sutured it immediately. No abdominal was found to be abnormal in terms of computed tomography scan after the operation.

\section{Postoperative Complications}

Postoperative complications such as wound infection, reverse ejaculation and psoas major hematoma did not occur in the patients. The main postoperative complications can be divided into the following categories.

\section{Nerve Injury}

There were two cases (3.2\%) of left sympathetic nerve injury which recovered after 1 week of follow-up. two cases (3.2\%) of contralateral nerve root injury were caused by the compression of contralateral nerve root by fusion cage. They recovered after posterior nerve root decompression and pedicle screw fixation. Three cases (3.1\%) had transient left quadriceps weakness, which recovered 5 days after operation. One case $(1.0 \%)$ had transient left psoas weakness, and the patient was successfully treated one week after conservative therapy. There were three cases (3.1\%) of pain in left thigh and those patients of recovered 5 days after surgery.

\section{Cage-related Complications}


Cage-related complications are also the most common complications of stand-alone OLIF. The criteria for evaluating the cage subsidence was as follows: in lateral X-ray of lumbar spine, the sinking height of cage accounted for $0 \%-24 \%$ of the height of fusion cage, $25 \%-49 \%$ of grade I, $50 \%-74 \%$ of grade II and $75 \%-100 \%$ of grade III. In this study, 10 cases $(15.9 \%)$ suffered from cage subsidence, all of which were grade 0 . No second-stage posterior internal fixation was performed. During the follow-up, no recurrence of symptoms was found. One patient $(1.6 \%)$ had cage displacement after the operation, and the symptoms were relieved after posterior pedicle screw fixation and decompression.

\section{Other Complications}

One patient (1.6\%) had subcutaneous pneumatosis, after 4 weeks of follow-up, subcutaneous pneumatosis was absorbed.

\section{Discussion}

The intervertebral disc height will be lost after the degeneration of the intervertebral disc, causing the instability of the spine and the relaxation of the ligamentum flavum, which could be regarded as one of the most important mechanism leading to the stenosis of the lumbar spinal canal ${ }^{[12]}$. At present, there are direct decompression and fusion operations such as PLIF and TLIF and indirect decompression surgery such as D/XLIF for the treatment of lumbar spinal stenosis. There are also cases of OLIF for the treatment of lumbar spinal stenosis reported in the literature ${ }^{[13-14]}$. To date, this is the first report of stand-alone OLIF in the treatment of lumbar spinal stenosis.

In this study, the effect of indirect neural decompression was evaluated by comparing the preoperative, postoperative and the final follow-up of $\mathrm{DH}$ and $\mathrm{FH}$ in terms of $\mathrm{X}$-ray imaging. The results showed that both postoperative $\mathrm{DH}$ and $\mathrm{FH}$ were significantly higher than those of preoperation, which was contributed to the large OLIF cage. What's more, we can clearly observed the excision of herniated intervertebral disc as well as the stretching and thinning of the ligamentum flavum through MRI image (Fig. 6). It indicated that the indirect spinal canal decompression of OLIF is achieved by increasing the height of intervertebral disc and intervertebral foramen and restoring the length and tension of ligamentum flavum. Previous literature also showed similar results. Lin et al ${ }^{[15]}$ measured 25 patients who received OLIF with and without posterior internal fixation and found that average DH was significantly restored from $8.97 \mathrm{~mm}$ preoperatively to $13.44 \mathrm{~mm}$ postoperatively, and $\mathrm{FH}$ was significantly increased from a mean of $19.68 \mathrm{~mm}$ before surgery to $23.42 \mathrm{~mm}$ after surgery. In the authors' experience, the cage could be placed as far back as possible for patients with lumbar spinal stenosis so as to distract the intervertebral space to the greatest extent. On the contrary, the cage should be put forward for patients with lumbar spondylolisthesis, which is conducive to the reduction of spondylolisthesis. Something interesting was found that the results of both $\mathrm{DH}$ and $\mathrm{FH}$ decreased at the last follow-up compared to those of postoperation, but still higher than those before surgery, with statistical significance. We thought it was due to cage subsidence without posterior pedicle screw fixation. 
Complications played an important role in the clinical outcomes. In this study, there were a total of 27 cases of complications in the intraoperative and postoperative stages, including 8 patients with two or more combined complications. Therefore, there were actually 19 patients, and the complication incidence was $30.2 \%$, which was similar to those reported in the previous literature ${ }^{[6,16]}$. Herein, stand-alone OLIF complications can be divided into two parts, approach-related complications and cage-related complications included. There were 13 cases were approach-related complications, with a rate of $20.6 \%$, including leg numbness $17.4 \%$ (11/63), vascular injury $1.6 \%(1 / 63)$ and peritoneal injury $1.6 \%(1 / 63)$. The similar result was also reported by Abe et al ${ }^{[17]}$ who reviewed 155 patients received OLIF and found that approach-related complications were $16.1 \%$. However, the most remarkable complications were cagerelated complications for stand-alone technology. 10 patients (15.9\%) with cage subsidence and 1 patient $(1.6 \%)$ with cage displacement were observed. In a recent study, Zeng et al ${ }^{[18]}$ found that the early complication incidence of stand-alone OLIF group was $36.26 \%$, which was higher than that of OLIF combined posterior pedicle screw fixation group, being $29.86 \%$. This was owing to the higher incidence of cage sedimentation in the stand-alone OLIF group than in the OLIF combined posterior fixation group. Therefore, some researchers advised posterior internal fixation in single stage for all patients undergoing the OLIF procedure because they worried about the limited relief or aggravation of clinical symptoms due to cage subsidence. As far as I'm concerned, stand-alone OLIF was sufficient for selected patients. It was believed that large cage could not only provide a more effective biological environment for the fusion process but also reduce the incidence of cage subsidence. Besides, the OLIF cage with 6 degrees of lordosis angle we used in the operation increased the lumbar lordosis from $38.0^{\circ} \pm 15.6^{\circ}$ preoperative to $42.7^{\circ} \pm 13.0^{\circ}$ at the final follow-up. In addition, Hresko et al ${ }^{[19]}$ indicated that the recovery of lumbar lordosis was beneficial to increase the tension of anterior longitudinal ligament, which helped increasing the rate of spine fusion. Previous studies also exhibited the efficiency of stand-alone technology. Nitin Agarwal et al ${ }^{[20]}$ investigated 55 patients with stand-alone LLIF with a ten year follow up and proved that stand-alone LLIF was a safe and effective surgical method. Solid arthrodesis and improvements in clinical symptoms using stand-alone XLIF were observed over $80 \%$ of patients in another study ${ }^{[21]}$. Zhang et al ${ }^{[22]}$ analyzed a total of 45 segments in 22 patients using the OLIF procedure and found cage subsidence occurring in 15 fused segments. However, symptoms in all patients had been alleviated and pain were improved at the final follow-up. So he considered stand-alone OLIF as a safe and effective method for patients who require one-and two-level interbody fusion with the diagnosis excluding lumbar spondylolisthesis, degenerative scoliosis and spondylolysis. In the opinion of Schiffman, there was no direct relationship between the radiographic cage subsidence and clinical efficacy ${ }^{[23]}$. Ahmadian et al ${ }^{[24]}$ investigated 59 patients with stand-alone LLIF and found that $9 \%$ of the patients remained clinically asymptomatic even if the their cage subsidence were high grade ( $\geqq$ grade II). It was recommended that patients with osteoporosis, body mass index more than $30 \mathrm{~kg} / \mathrm{m}^{2}$ or intraoperative endplate damage needed additional posterior internal fixation ${ }^{[18]}$.

There are still some limitations in this study. Firstly, the cases were selected retrospectively. Secondly, the duration of follow-up was short. Further studies are required to ascertain the indications for single-stage 
or multistage internal fixation and posterior decompression.

\section{Conclusion}

We evaluated the clinical and radiographic outcomes of stand-alone OLIF for patients with spinal stenosis. Significant improvements in disc height and FH were found after stand-alone OLIF with indirect decompression. The results also proved that VAS score and ODI had been improved. It seems that standalone OLIF maybe used as an alternative treatment in selected patients with lumbar spinal stenosis.

\section{Abbreviations}

OLIF Oblique lumbar interbody fusion

VAS Visual Analogue Scale

ODI Oswestry Disability Index

DH Disc height

FH Foraminal height

PLIF Posterior lumbar interbody fusion

TLIF Transforaminal lumbar interbody fusion

ALIF Anterior lumbar interbody fusion

D/XLIF Extreme/direct lateral interbody fusion

LL Lumbar lordosis

\section{Declarations}

\section{Ethics approval and consent to participate}

Ethical approval for this prospective study was obtained from the Ethics Committee of Wannan Medical college affiliated Yijishan Hospital. All the patients have provided their written informed consent to participate in this study.

\section{Consent to publish}

All participants gave written informed consent for publication of the results.

\section{Availability of data and materials}


The datasets used in the current study are available from the corresponding author

\section{Competing interests}

The authors declare that they have no competing interests.

\section{Funding}

The study was supported by the The National Natural Science Foundation of China (81572185 (Prof. Xu), 81702158 (Doctor. Liu)). Funding of "Peak" Training Program and "Panfeng" Innovation Team Project for Scientifc Research of Yijishan Hospital, Wannan Medical College (GF2019T02 (Prof. Xu), GF2019G07 (Doctor. Liu), GF2019G12 (Doctor. Xiao), PF2019007 (Prof. Xu)).

\section{Authors' Contributions}

$\mathrm{CL}$ and QZ carried out project conceptualization, data collection, and drafted the manuscript. YZ, LX and XG performed the statistical analysis and participated in its design. HX participated in the project conceptualization and the guarantor of the article. All authors read and approved the final manuscript.

\section{Acknowledgements}

None.

\section{References}

1. Siebert E, PrüssH, Klingebiel R, et al. Lumbar spinal stenosis: syndrome, diagnostics and treatment[J]. Nat Rev Neurol, 2009, 5(7):392-403

2. Schller K, Alimi M, Cong GT, et al. Lumbar Spinal Stenosis Associated With Degenerative Lumbar Spondylolisthesis: A Systematic Review and Meta-analysis of Secondary Fusion Rates Following Open vs Minimally Invasive Decompression[J]. Neurosurgery, 2017;80(3):355-367.

3. Mobbs R J, Phan K, Malham G, et al. Lumbar interbody fusion: techniques, indications and comparison of interbody fusion options including PLIF, TLIF, MI-TLIF, OLIF/ATP, LLIF and ALIF[J]. Journal of Spine Surgery, 2015, 1(1): 2.

4. Than K D, Wang A C, Rahman S U, et al. Complication avoidance and management in anterior lumbar interbody fusion[J]. Neurosurgical focus, 2011, 31(4): E6.

5. Lykissas M G , Aichmair A, Hughes A P, et al. Nerve injury after lateral lumbar interbody fusion: a review of 919 treated levels with identification of risk factors[J]. The Spine Journal, 2014, 14(5):749758

6. Ohtori S, Orita S, Yamauchi K, et al. Mini-open anterior retroperitoneal lumbar interbody fusion: oblique lateral interbody fusion for lumbar spinal degeneration disease[J]. Yonsei medical journal, 2015, 56(4): 1051-1059. 
7. Castellvi A E, Nienke T W, Marulanda G A, et al. Indirect decompression of lumbar stenosis with transpsoas interbody cages and percutaneous posterior instrumentation[J]. Clinical Orthopaedics and Related Research ${ }^{\circledR}, 2014,472(6)$ : 1784-1791.

8. Fujibayashi S, Hynes R A, Otsuki B, et al. Effect of indirect neural decompression through oblique lateral interbody fusion for degenerative lumbar disease[J]. Spine, 2015, 40(3): E175-E182.

9. Lykissas M G , Aichmair A, Hughes A P, et al. Nerve injury after lateral lumbar interbody fusion: a review of 919 treated levels with identification of risk factors[J]. The Spine Journal, 2014, 14(5):749758.『

10. Silvestre C, Mac-Thiong J M, Hilmi R, et al. Complications and Morbidities of Mini-open Anterior Retroperitoneal Lumbar Interbody Fusion: Oblique Lumbar Interbody Fusion in 179 Patients[J]. Asian Spine Journal, 2012, 6(2):89-97.

11. Schizas C, Theumann N, Burn A, et al. Qualitative grading of severity of lumbar spinal stenosis based on the morphology of the dural sac on magnetic resonance images[J]. Spine, 2010, 35(21): 19191924.]

12. Karantanas $A \mathrm{H}$, Zibis $A \mathrm{H}$, Papaliaga $\mathrm{M}$, et al. Dimensions of the lumbar spinal canal: variations and correlations with somatometric parameters using CT[J]. European radiology, 1998, 8(9): 1581-1585.

13. Sato J, Ohtori S, Orita S, et al. Radiographic evaluation of indirect decompression of mini-open anterior retroperitoneal lumbar interbody fusion: oblique lateral interbody fusion for degenerated lumbar spondylolisthesis[J]. European Spine Journal, 2017, 26(3): 671-678.

14. Fujibayashi S, Hynes R A, Otsuki B, et al. Effect of indirect neural decompression through oblique lateral interbody fusion for degenerative lumbar disease[J]. Spine, 2015, 40(3): E175-E182.

15. Lin G X, Akbary K, Kotheeranurak V, et al. Clinical and Radiologic Outcomes of Direct Versus Indirect Decompression with Lumbar Interbody Fusion: A Matched-Pair Comparison Analysis[J]. World neurosurgery, 2018, 119: e898-e909.

16. Mehren C, Mayer HM, Zandanell C, Siepe CJ, Korge A. The oblique anterolateral approach to the lumbar spine provides access to the lumbar spine with few early complications. Clin Orthop Relat Res, 2016, 474: 2020-2027》

17. Abe K, Orita S, Mannoji C, et al. Perioperative complications in 155 patients who underwent oblique lateral interbody fusion surgery: perspectives and indications from a retrospective, multicenter survey[J]. Spine, 2017, 42(1): 55-62.

18. Zeng Z, Xu Z, He D, et al. Complications and prevention strategies of oblique lateral interbody fusion technique[J]. Orthopaedic surgery, 2018, 10(2): 98-106.

19. Hresko MT, Labelle H, Roussouly P, Berthonnaud E. Classification of high-grade spondylolistheses based on pelvic version and spine balance: possible rationale for reduction. Spine (Phila Pa 1976). 2007. 32(20):2208-13.

20. Agarwal N, Faramand A, Alan N, et al. Lateral lumbar interbody fusion in the elderly: a 10-year experience: Presented at the 2018 AANS/CNS Joint Section on Disorders of the Spine and Peripheral Nerves[J]. Journal of Neurosurgery: Spine, 2018, 29(5): 525-529. 
21. Youssef J A, McAfee P C, Patty C A, et al. Minimally invasive surgery: lateral approach interbody fusion: results and review[J]. Spine, 2010, 35(26S): S302-S311.

22. Zhang $C$, Wang $K$, Jian F, et al. Efficacy of Oblique Lateral Interbody Fusion in Treatment of Degenerative Lumbar Disease[J]. World neurosurgery, 2019, 124:e17-e24.

23. Schiffman M, Brau S A, Henderson R, et al. Bilateral implantation of low-profile interbody fusion cages: subsidence, lordosis, and fusion analysis[J]. The Spine Journal, 2003, 3(5): 377-387.

24. Ahmadian A, Bach $K$, Bolinger $B$, et al. Stand-alone minimally invasive lateral lumbar interbody fusion: multicenter clinical outcomes[J]. Journal of Clinical Neuroscience, 2015, 22(4): 740-746.

\section{Tables}

Due to technical limitations, Tables 1-2 are provided in the Supplementary Files section.

\section{Figures}
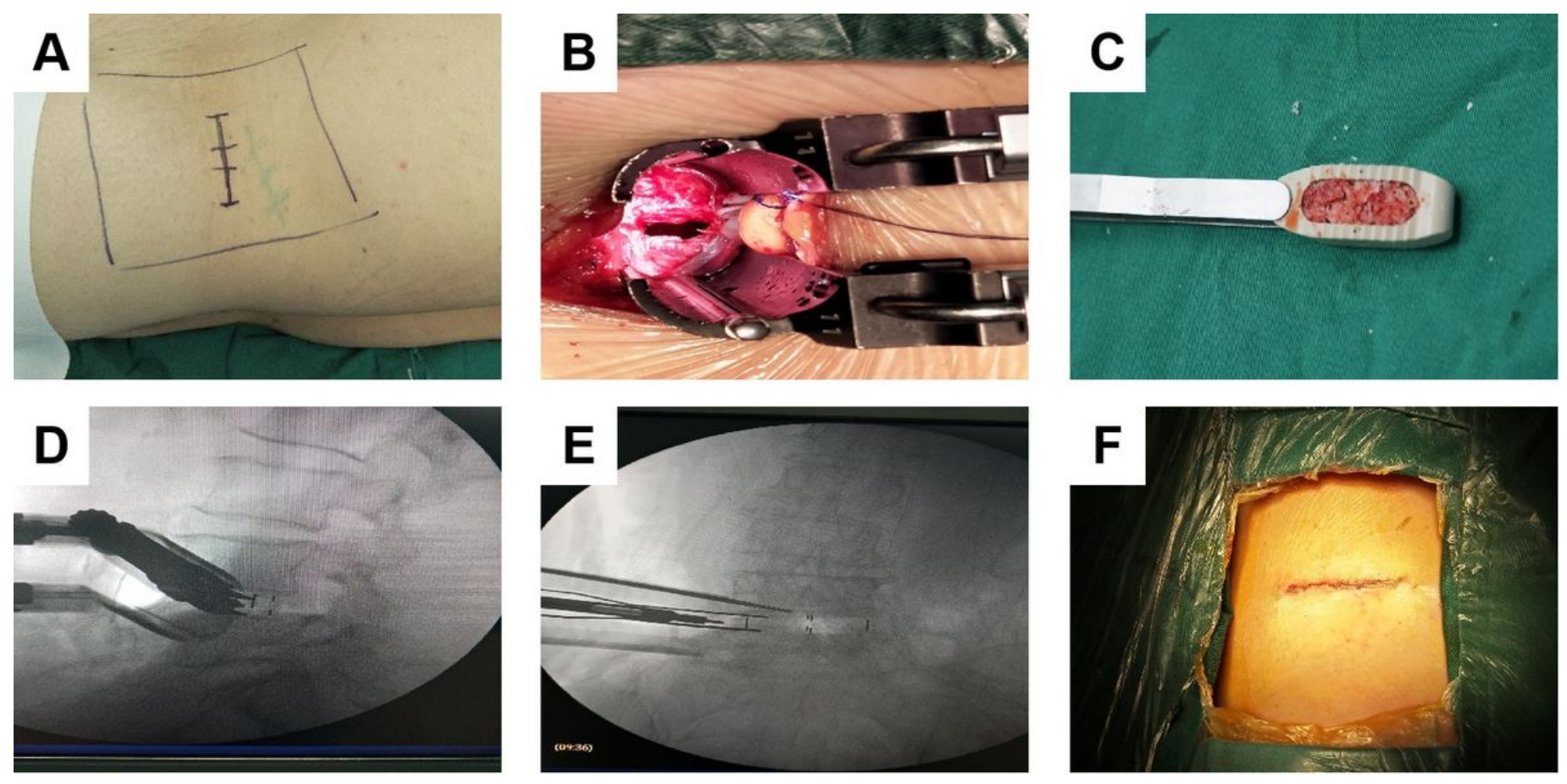

\section{Figure 1}

Procedure of stand-alone OLIF operation. (A) The skin incision was marked with the help of C-arm before the surgery. (B) Retractor for OLIF used after dilatation. (C) A clydesdale cage was filled with allograft bone. $(D, E)$ The $X$-ray of cage implantation in lateral direction. $(F)$ Skin closure was performed. 


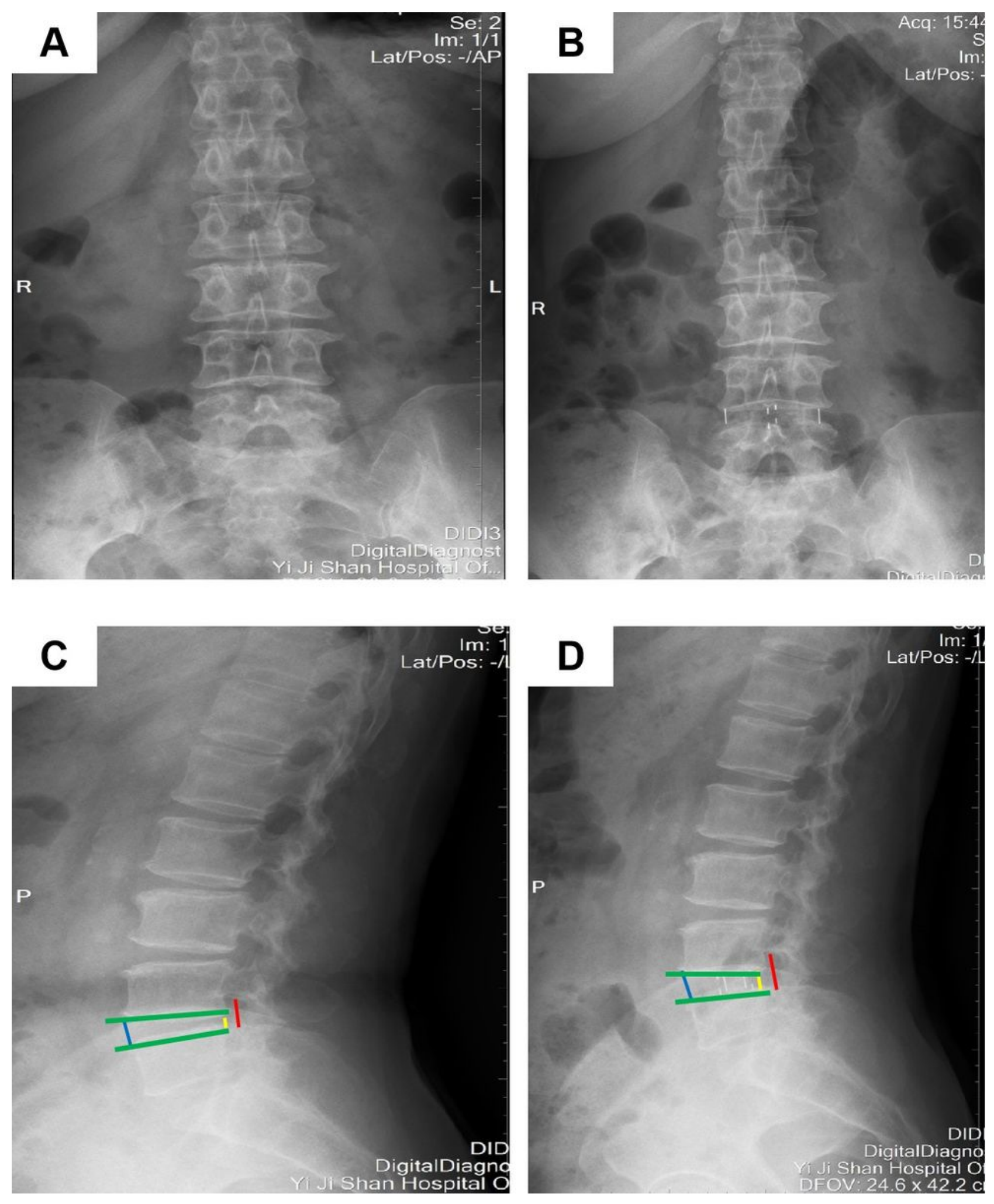

\section{Figure 2}

The X-ray before surgery $(A, C)$ and after surgery $(B, D)$. The distance between the anterior/posterior edges of the upper vertebrae and the end plate of the lower vertebrae is called the anterior/posterior $\mathrm{DH}$. The average value of anterior disk height (marked blue) and posterior disc height (marked yellow) is DH (C,D). The FH was measured in the lateral X-ray image. The length between the upper and lower edges (marked red) is $\mathrm{FH}(\mathrm{C}, \mathrm{D})$. 

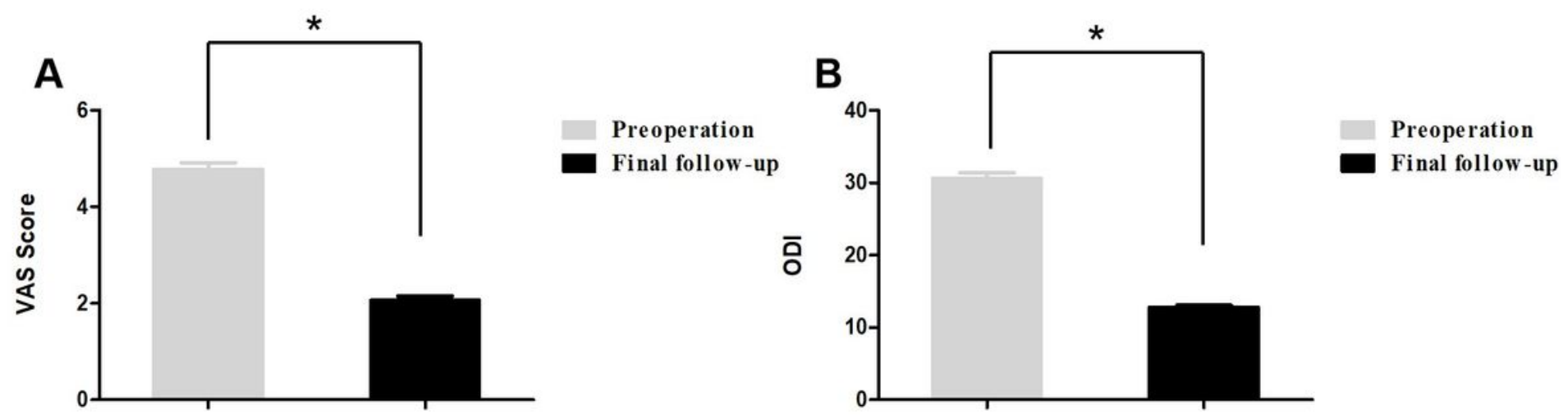

Figure 3

VAS scores (A) and ODI (B) were significantly improved at the final follow-up compared with preoperation $(p<0.01)$.
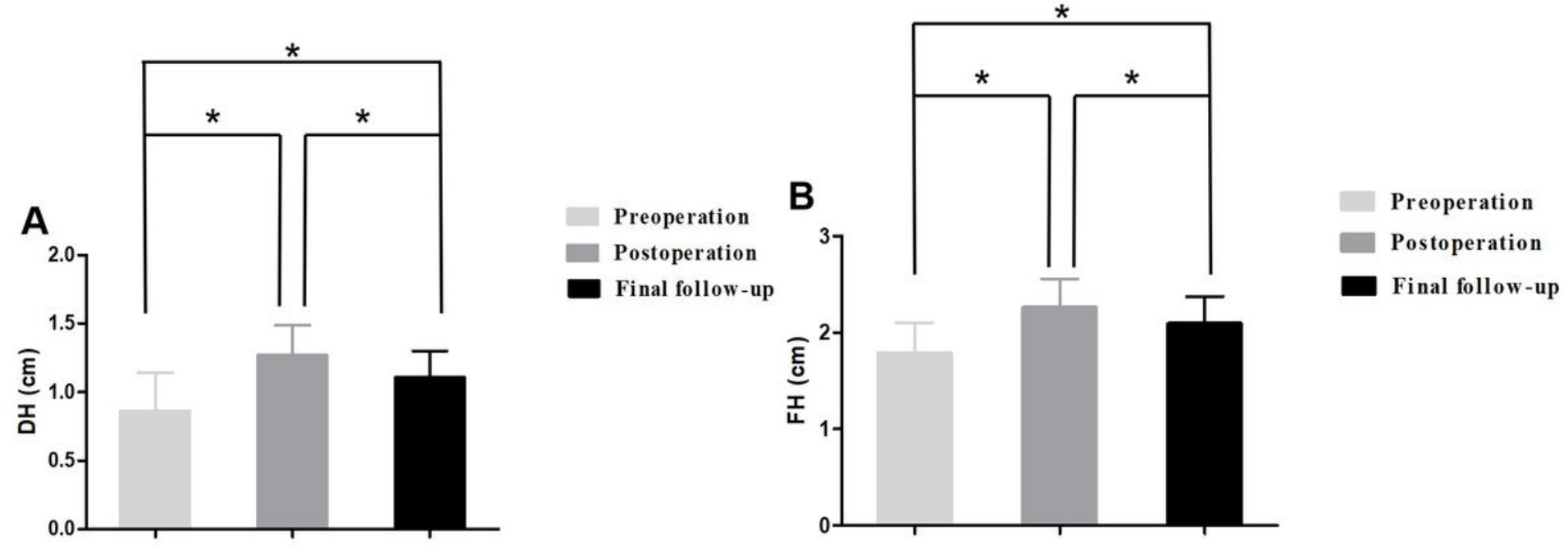

Figure 4

The $\mathrm{DH}(\mathrm{A})$ increased from $0.9 \pm 0.3 \mathrm{~cm}$ before the operation to $1.3 \pm 0.2 \mathrm{~cm}$ after the operation, and the final follow-up was $1.1 \pm 0.2 \mathrm{~cm}(p<0.01)$. The $F H(B)$ increased from preoperative $1.7 \pm 0.3 \mathrm{~cm}$ to postoperative $2.3 \pm 0.3 \mathrm{~cm}$ and decreased to $2.1 \pm 0.3 \mathrm{~cm}$ at the final follow-up $(p<0.01)$. 


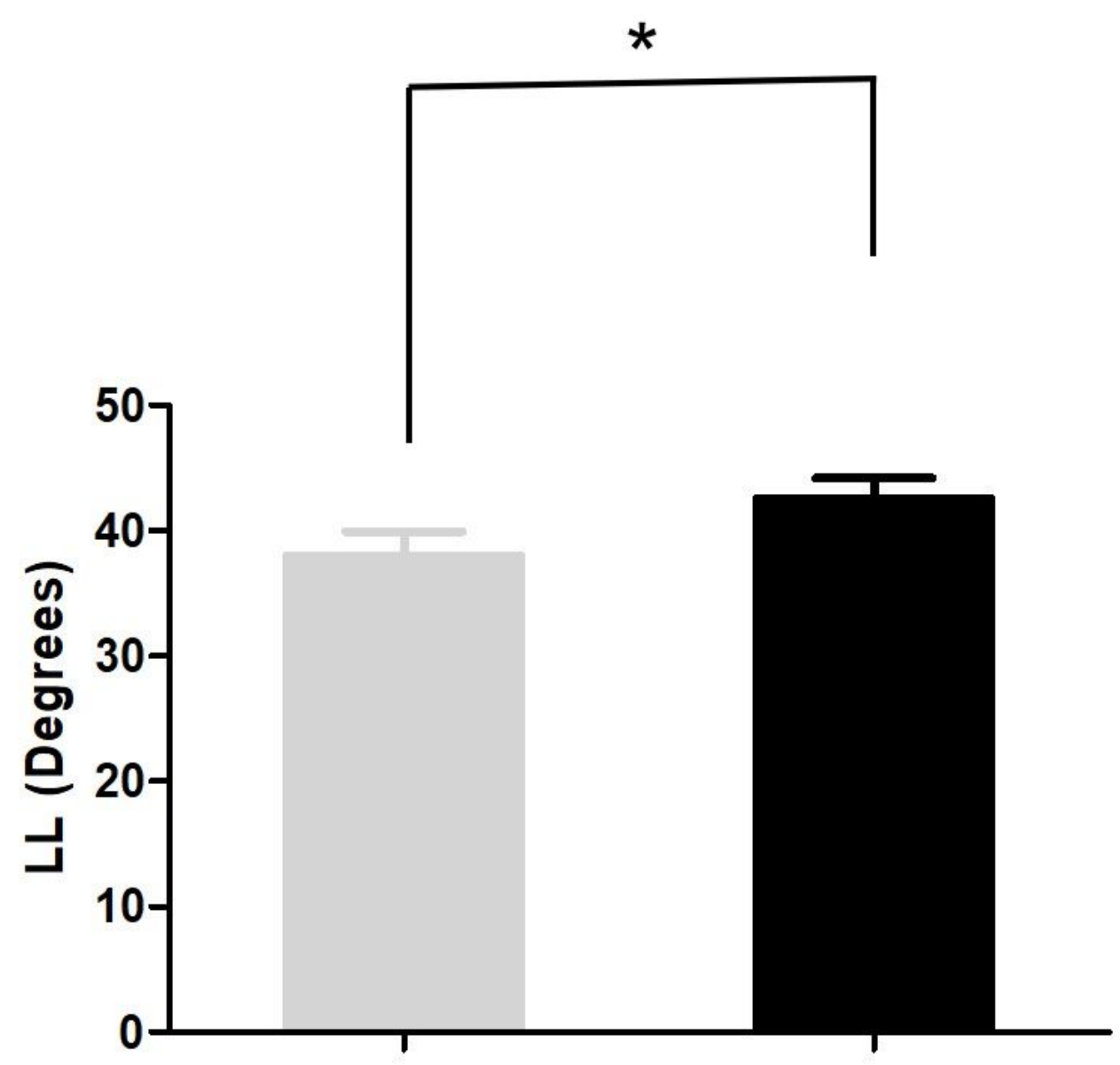

\section{Preoperation} Ginal follow-up

\section{Figure 5}

The lumber lordosis improved from $38.0^{\circ} \pm 15.6^{\circ}$ preoperatively to $42.7^{\circ} \pm 13.0^{\circ}$ at the final follow-up $(p<0.01)$. 

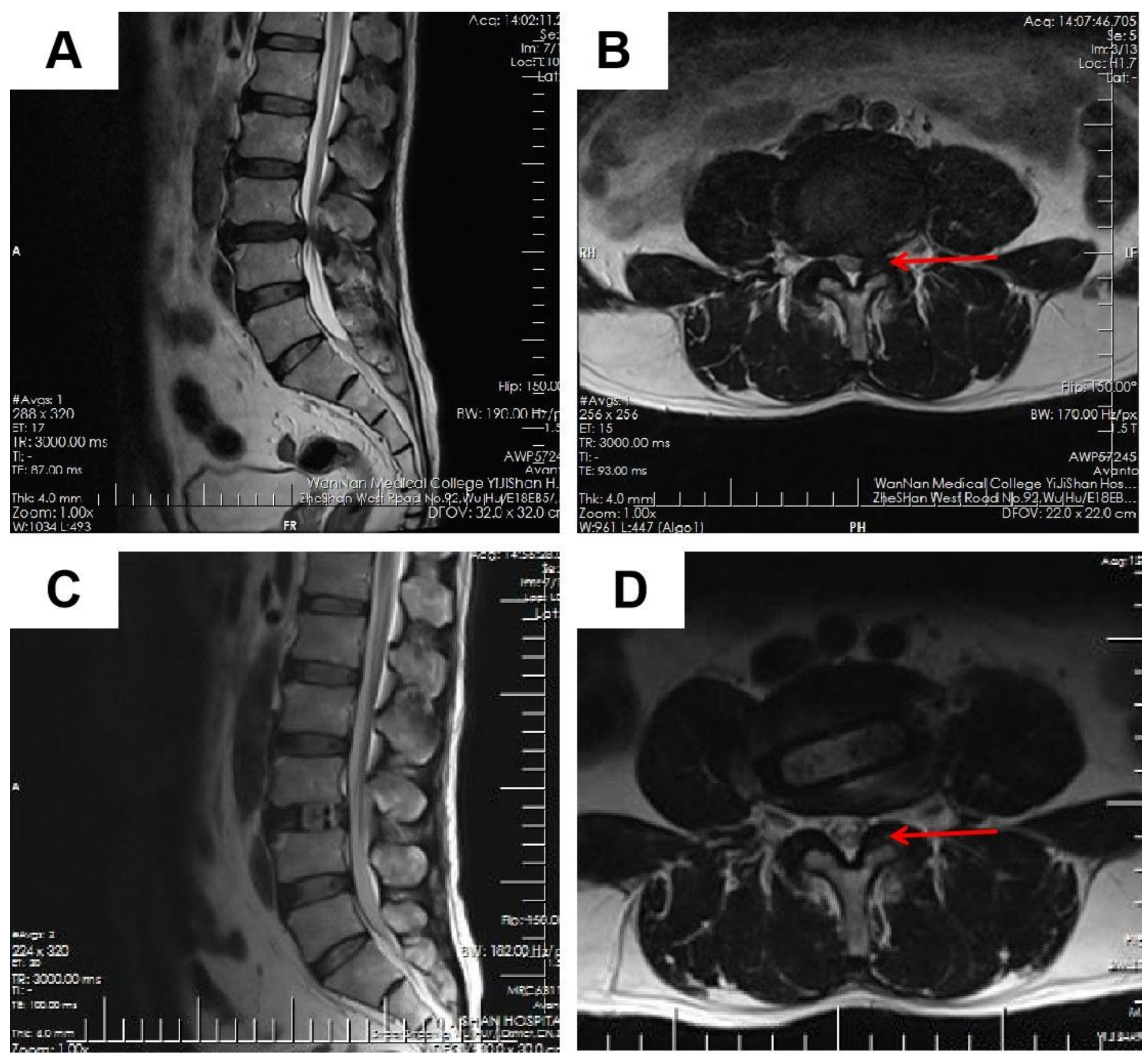

\section{Figure 6}

T2-weighted imaging before surgery $(A, B)$ and after surgery $(C, D)$. The excision of herniated intervertebral disc as well as the stretching and thinning of the ligamentum flavum were observed as the red arrow showed.

\section{Supplementary Files}

This is a list of supplementary files associated with this preprint. Click to download.

- Table.doc 